\title{
A comparative analysis of three typical crash tests results based on small overlap frontal collision accidents
}

\author{
Zhixin Liu*, Weijie Ma, Lei Lou and \\ Weidong Liu
}

China Automotive Technology and Research Centre Co., Ltd., Tianjin 300300, China

Email: liuzhixin@catarc.ac.cn

Email: maweijie@catarc.ac.cn

Email: loulei@catarc.ac.cn

Email: liuweidong@catarc.ac.cn

*Corresponding author

\begin{abstract}
With the promotion of collision safety technical regulations, the vehicle safety technology has achieved a leap-forward development, and the occupants have been well protected in various collision conditions. However, the statistical data indicate that serious accidental casualties are still present in frontal collision traffic accidents with small overlap ratios. One of the main reasons is that vehicles in the small overlap collisions did not have the energy absorption effect of the longitudinal beams and body structure, causing serious deformation of the passenger compartment and injuries to the occupants. In this paper, the characteristics of small overlap accidents were analysed based on the China traffic accident database. Then, actual vehicle crash tests were carried out by adopting three typical small overlap crash test methods (vehicle-tovehicle test method, IIHS test method and NHTSA test method), and in-depth analysis was conducted on the vehicle kinematics, collision strength (vehicle body acceleration and vehicle pulse index), occupant injuries, and vehicle body deformation in the crash tests. The results show that although the incidence of small overlap crash is low, the ratio of AIS3 + injury is very high, and the NTHSA test method is more similar to the vehicle-to-vehicle test than the IIHS test method.
\end{abstract}

Keywords: passive safety; traffic accident data; small overlap collisions; crash tests.

Reference to this paper should be made as follows: Liu, Z., Ma, W., Lou, L. and Liu, W. (2020) 'A comparative analysis of three typical crash tests results based on small overlap frontal collision accidents', Int. J. Vehicle Safety, Vol. 11, No. 3, pp.275-288.

This paper is a revised and expanded version of a paper entitled 'A comparative analysis of three typical crash tests results based on small overlap frontal collision accidents' presented at the 'International Symposium on Current Automotive Safety and Human Body Injury Modelling for Present and Future Mobility', Tianjin, China, 1-3 November 2018. 


\section{Introduction}

With the continued introduction of automotive safety standards and NCAP evaluation procedures, the safety technology of automotive collision in China has undergone remarkable development. The continuous development of automotive safety technology has greatly improved the crashworthiness and occupant protection capabilities of vehicles in frontal collisions. However, statistical data indicate that serious accidental casualties are still present in frontal collision traffic accidents with small overlap ratios. The reason is that vehicles in the small overlap collisions did not have the energy absorption effect of the longitudinal beams and body structure, causing serious deformation of the passenger compartment, and high crash strength was transmitted directly to the occupants, causing serious injuries to the occupants. As is shown in Figure 1, foreign accident statistics show that in a frontal collision, accident, similar to a tree crash or a car-to-car overlap collision accidents account for a relatively high proportion (Bean et al., 2009; United Nations Economic Commission for Europe, 2015; National Highway Traffic Safety Administration, 2009).

Figure 1 Small overlap impact traffic accident rate

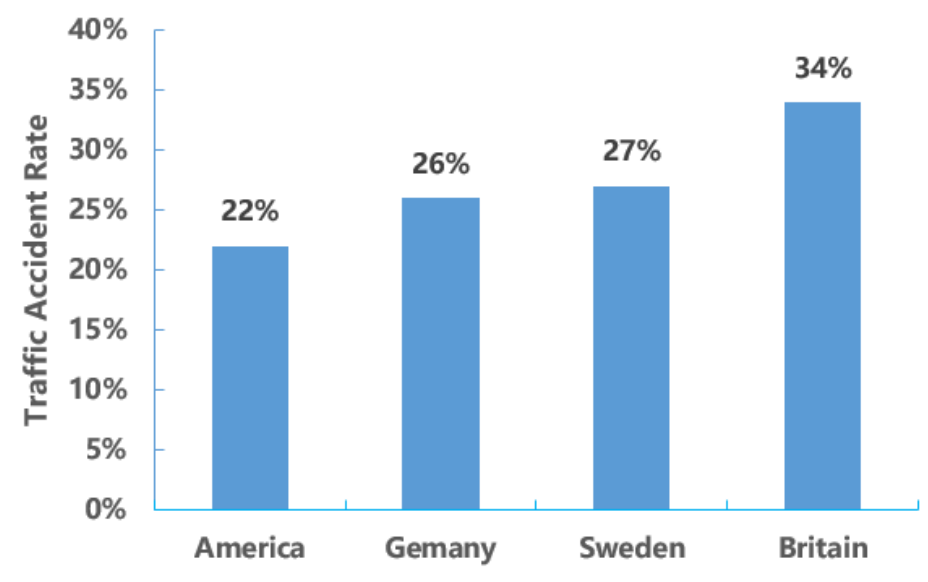

The USA small overlap collision accounts for $22 \%$ of the total frontal collisions, while that of Germany and Sweden, respectively, accounts for $26 \%$ and $27 \%$, and this figure is even higher in the UK, up to $34 \%$. Studies have shown that the common feature of these collisions is that the vehicle's front-end energy-absorbing and collision-resisting structure has little or no involvement. The front-end structure of the vehicle only collides with a very small overlap rate. As the energy-absorbing part does not play a role, it often brings a huge body structure into the cockpit and a strong lateral movement. At this time, the lower limbs and the head and neck of the occupants are injured due to this movement posture (Rudd et al., 2011; Ryuuji et al., 2015). NHTSA and IIHS conducted an in-depth study of such accidents based on the crashworthiness sample system in the US National Vehicle Accident Samples Database (Saunders et al., 2011; Sherwood et al., 2009). How to use a certain test method to the greatest degree to represent a small overlap collision and to test the protection of vehicle structure and occupant restraint system is a key research work in this field (James and Dan, 2013; Saunders et al., 2012). Later, the 
researchers also conducted comparative research on small overlap collision testing methods (Joyce et al., 2014) and a large amount of research on human injury assessment indicators in collision testing (Takhounts et al., 2013; Maika et al., 2015; Becky et al., 2015; Mueller et al., 2011). Some new methods of dealing with small overlap collision tests have also been proposed (Claudia et al., 2017; Dinesh et al., 2015). It can be seen that the evaluation of small overlap collision tests and their response methods are the key research contents of automotive safety in recent years.

At present, China is short of researches on small overlap collision test evaluation technology. Considering that this kind of collision test puts forward higher requirements on the safety of automobiles, the main research contents of this paper include the research on traffic accident statistical analysis methods and the data of the proportion and collision characteristics of small overlap collision accidents in China based on accident statistics analysis of domestic traffic. The test methods for small overlap collisions can be roughly divided into three forms, i.e., vehicle-to-vehicle collisions, car-to-rigid fixed barrier collisions and car-to-moving barrier. In this paper, through three real vehicle collision tests, the characteristics of the above three types of crash tests are analysed through four aspects: vehicle dynamic response, crash intensity, occupant injury and body deformation. It will be of great significance for the in-depth development of test evaluation methods and the formulation of standards and regulations in the future.

\section{Field data analysis based on China traffic accident database}

\subsection{The characteristic parameters of collision vehicles}

The characteristics of small overlap collision accidents mainly include three points: (1) The collision has a small overlap rate, generally less than $1 / 3$ of the vehicle width; (2) The front longitudinal beam of the main energy-absorbing components of the vehicle cannot play a corresponding role due to the low overlap rate; (3) Occasionally, the occupant will move obliquely in the event of a small overlap collision, resulting in the original restraint system failing to fully exert its protective effect. Therefore, when the accident data screening analysis is carried out in this paper, the definition of small overlapped collision accidents (as shown in Figure 2) is shown as follows: In the case of frontal collision, the initial collision load is concentrated on the outside of the longitudinal beam (about $25 \%$ of the vehicle width), and the objects are cars. The collision areas are at $1 / 4$ left and $1 / 4$ right of the vehicle, accounting for $56 \%$ and $44 \%$, respectively.

Figure 2 Definition of small overlap collision accidents

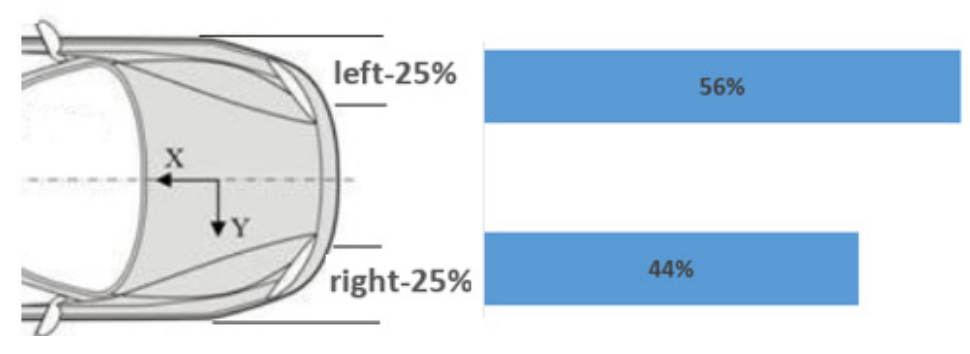


Figure 3 shows that the proportion of small overlap frontal collision accidents in China accounts for $27 \%$. From the perspective of the degree of accidental injuries, serious injuries and the death rate of $1 / 3$ overlap are $17 \%$ and $11 \%$, respectively, while severe injuries and the death rate of $1 / 4$ overlap are $6 \%$ and $15 \%$, respectively, which are much higher than other overlap collision forms.

Figure 3 The proportion of small overlap accidents in China

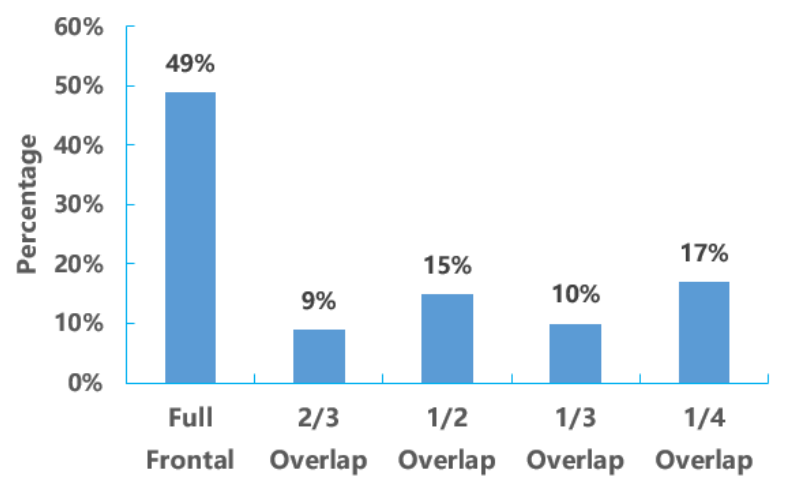

As is shown in Figure 4(a), the vehicle speed from a small overlap collision has a wide distribution range. $93 \%$ of vehicles in collision reach a speed over $40 \mathrm{~km} / \mathrm{h}$, while vehicles at the speed between $60 \mathrm{~km} / \mathrm{h}$ and $80 \mathrm{~km} / \mathrm{h}$ have the highest ratio of collisions, accounting for approximately $35 \%$. The proportion of cars moving over $80 \mathrm{~km} / \mathrm{h}$ also reaches $37 \%$. Therefore, it can be seen that the vehicle speed in the small overlap collision accident is higher than other accident types. Figure 4(b) shows mean value of speed is $59.8 \mathrm{~km} / \mathrm{h}$, and the standard deviation is $18.73 \mathrm{~km} / \mathrm{h}$. The average speed of vehicles in small overlap collision accidents is approximately $62.4 \mathrm{~km} / \mathrm{h}$.

As the front longitudinal beam does not have a good effect of collision energy absorption in the small overlap collision accidents, the longitudinal deformation of the collision area is significantly larger than other collision types, which endangers the safety of the occupants. According to the statistical analysis of longitudinal deformation of vehicle collision, as is shown in Figure 5, the longitudinal deformation of the vehicle distributed in $30-60 \mathrm{~cm}$ accounts for a large part, up to $44.2 \%$; deformation greater than $60 \mathrm{~cm}$ accounts for $44.2 \%$. From the perspective of vehicle structure, when the deformation is more than $60 \mathrm{~cm}$, it has obviously threatened the living space of the occupant's cabin, resulting in the occupant losing the necessary living space, that is, the damage is caused by squeezing. At the same time, the vehicle is rotating, and the occupant in the rotation process has a strong contact with the side parts of the vehicle, thus causing injury. 
Figure 4 Collision speed statistics in small overlap accidents

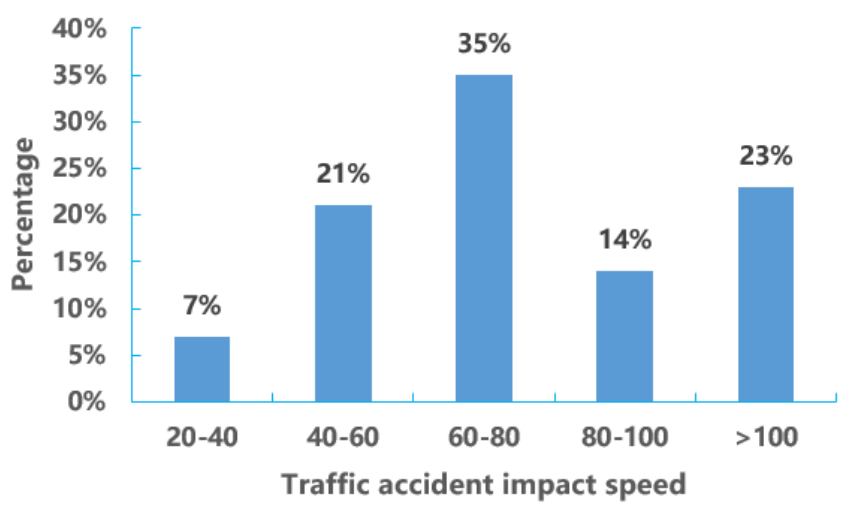

(a)

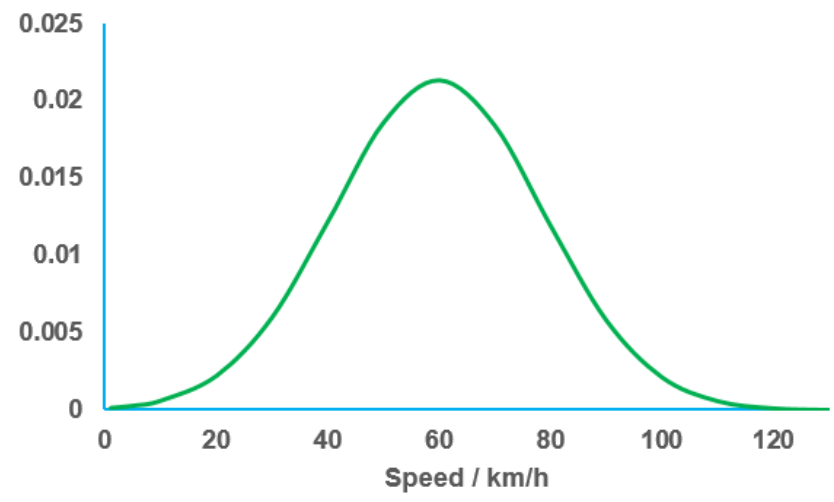

(b)

Figure 5 Statistics on longitudinal deformation of vehicles in small overlap collision

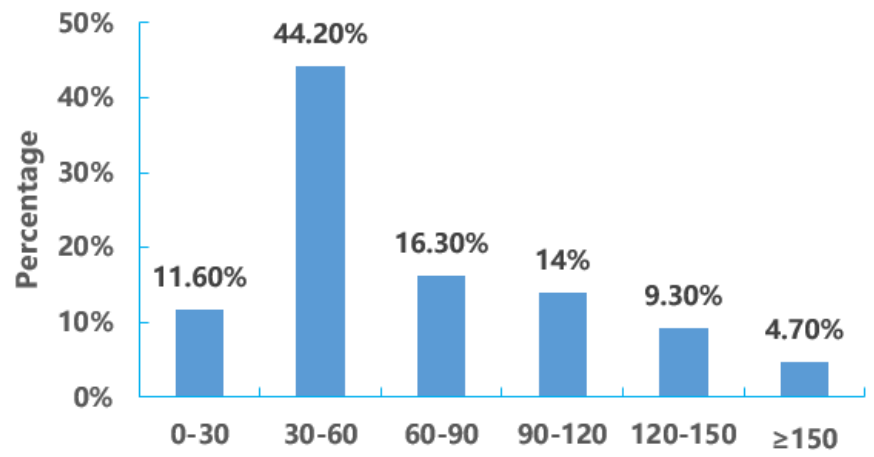

Longitudinal deformation/ cm 


\subsection{Occupants injuries of small overlap accidents}

According to the statistical analysis of the injuries in small overlap collision accidents, small overlap collision accidents cause relatively bad injuries to the occupants' head, face, and chest. In the distribution of all injuries, the number of head and facial injuries is 56 and it accounts for $49.6 \%$ of all injuries. The number of thoracic injury is 21 , accounting for $18.6 \%$, as is shown in Figure 6; in the statistical AIS3+ (severe damage level and above) injuries, the maximum number of head and face injuries is 14 , accounting for $61 \%$ of AIS3+injuries, as is shown in Figure 7. According to the statistics of the degree of injury in each injured area, it is found in the small overlap collision accidents that the proportion of injuries and the damage severity in the head and the face and the chest area of the occupants is significantly higher than those of other injuries.

Figure 6 Occupant injury distribution

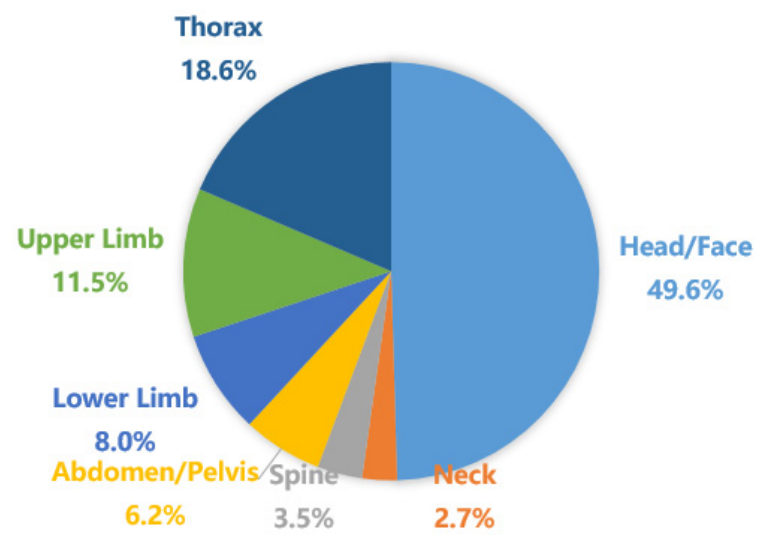

Figure 7 AIS3+ injury distribution of occupant

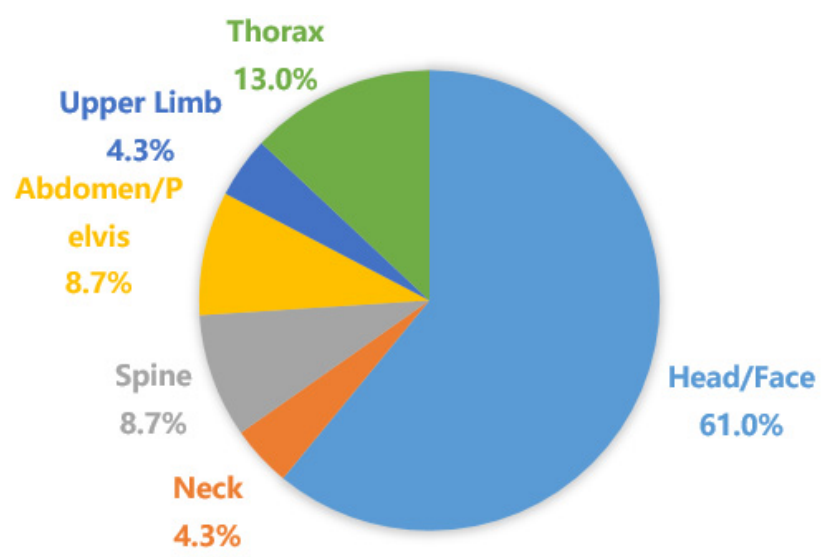

The statistical analysis of vehicle collision speeds in small overlap collision accidents indicates that in the accidents involving occupant death, the collision speed is greater than $35 \mathrm{~km} / \mathrm{h}$, and when the collision speed ranges from $35 \mathrm{~km} / \mathrm{h}$ to $75 \mathrm{~km} / \mathrm{h}$, the injury 
degree and proportion of the vehicle personnel is the highest. Among them, the proportion of minor injuries reaches $20.2 \%$, that of serious injuries is $7.4 \%$, and the death rate is also up to $4.3 \%$, as is shown in Figure 8 . In addition, it is found through comparative analysis that when the collision speed exceeds $75 \mathrm{~km} / \mathrm{h}$, the proportion of people who have suffered serious injuries and death increases, so does the degree of injury to the occupant.

Figure 8 Correlation of collision speed and occupant injury

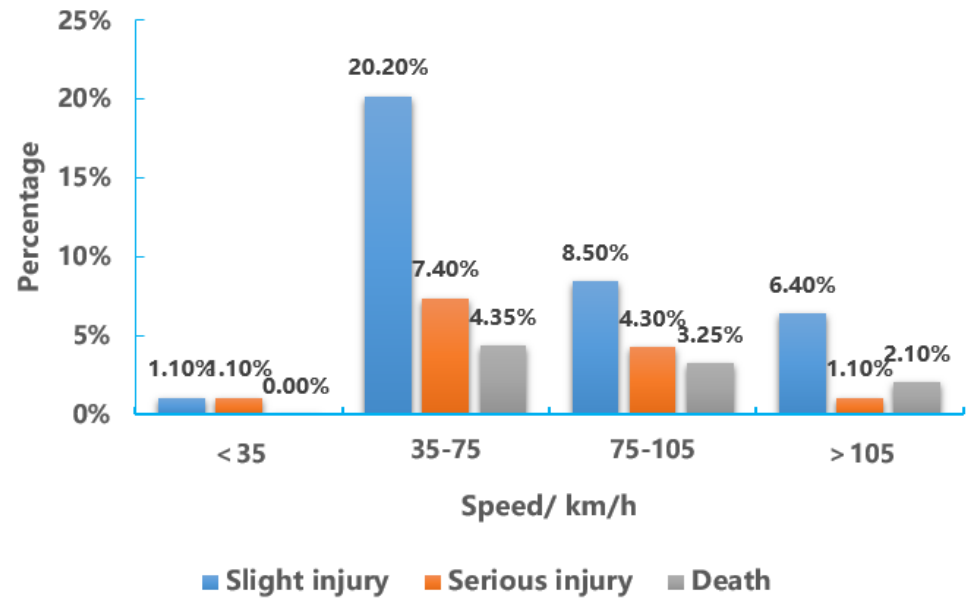

As shown in Figure 9, when the collision position is on the left side of the vehicle, the degree of driver's injury is significantly greater than that when the collision position is on the right side of the vehicle, especially the proportion of serious injury and death. It can be seen that in the case of a collision on the left side of the vehicle, the proportion of the driver suffering from serious injury is more than 6 times that in the case of a collision on the right side of the vehicle, while the proportion of the driver dying in the case of a collision on the left side is nearly 3 times that in the case of a collision on the right side of the vehicle.

Figure 9 Correlation of collision position and occupant injury

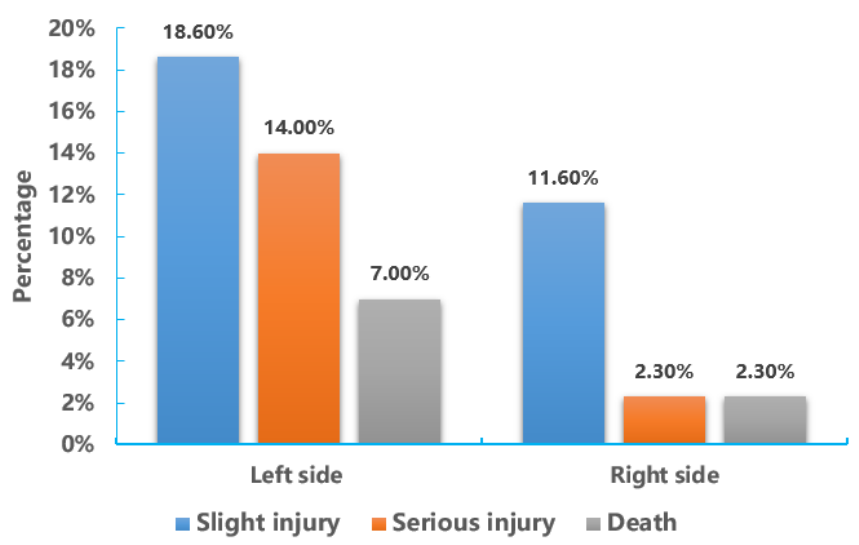


The statistical results of traffic accidents show that when there is a small overlap collision, the vehicle body will rotate around the $Z$-axis to a certain extent, of which $98 \%$ of the vehicle rotation angle is less than $90^{\circ}$. As shown in Figure 10, when the rotation angle of the vehicle is less than $90^{\circ}$ after collision, the proportions of slight injury, serious injury and death in the vehicle are $30.2 \%, 9.3 \%$ and $4.7 \%$, respectively, while when the rotation angle of the vehicle is greater than or equal to $90^{\circ}$, the proportion of death in the vehicle is $2.3 \%$. It can be seen that when the vehicle rotates more than $90^{\circ}$ in the collision, the risk to passengers in the vehicle is extremely high.

Figure 10 Correlation of vehicle body rotation and occupant injury

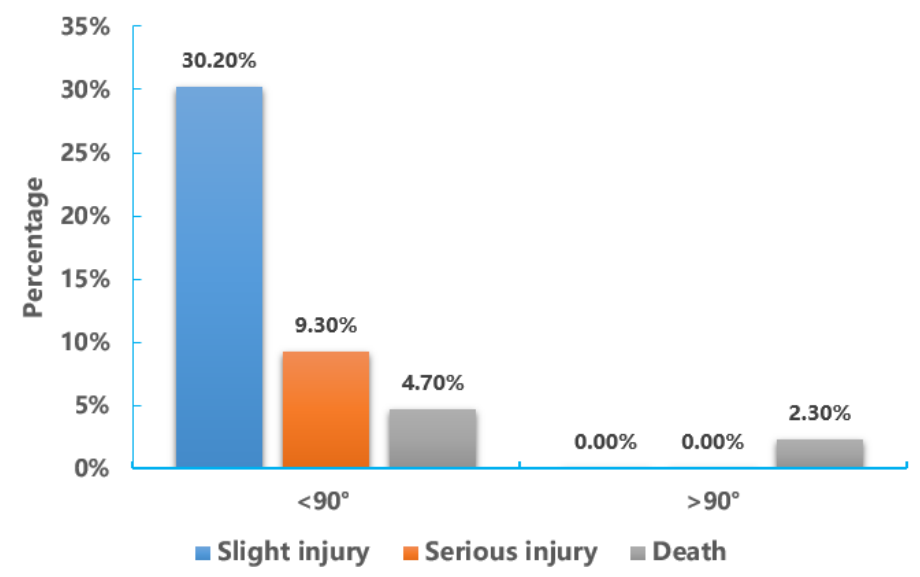

\section{Crash test methods}

From the characteristics of actual traffic accidents, there are three types of collisions in small offset collisions, that is, the vehicle-to-fixed objects (trees, columns, etc.), vehicleto-vehicle linear collisions, and vehicle-to-vehicle diagonal collisions. At present, the test methods for small overlap collisions are roughly divided into three types: vehicle-tovehicle collisions, vehicle-to-rigid fixed barriers, and MDB-impacted stationary vehicles. The collision speeds, overlap rates, and collision angles are also different. The important parameters are closely related to the actual road traffic injuries and distribution in each country. Therefore, it is imperative to develop small overlap collision test methods suitable for China's traffic characteristics in light of actual road traffic conditions in China.

\subsection{Test method of vehicle-to-vehicle small offset collision}

The vehicle-to-vehicle test program adopts the internationally popular vehicle-to-vehicle small offset collision test method. Both vehicles are moving toward each other at a speed of $56 \mathrm{kph}$. The approach angle is $15^{\circ}$ and the overlap ratio is $25 \%$. The vehicle-to-vehicle collision assessment method is based on the existing dummy damage, and refers to the NCAP evaluation method for the occupant. There is no clear performance parameter index for the vehicle body and restraint system. 


\subsection{Test method of IIHS small offset collision}

The specific collision pattern of IIHS is: the vehicle hits a rigid barrier $(1524 \mathrm{~mm}$ in height and $150 \mathrm{~mm}$ in radius) at a speed of $(64.4 \pm 1) \mathrm{km} / \mathrm{h}$, and the vehicle-barrier overlap rate is $25 \%$. Place a Hybrid III $50 \%$ male dummy to measure dummy damage.

\subsection{Test method of small offset collision of NHTSA}

The final test plan of NHTSA is adopted in this study, that is, the collision area between the vehicle and the head is $35 \%$, the test vehicle is stationary, the head of the car has a certain deflection angle, the impact inclination angle is $15^{\circ}$, and the test speed is $90 \mathrm{~km} / \mathrm{h}$. The Hybrid III 50\% male dummy is used to measure dummy damage.

\section{Comparison of test result}

Regardless of the small offset rigid barrier test of IIHS or the moving barrier angle collision test of NHTSA, these two types of experimental methods essentially appear in the actual traffic accidents on the condition of the small offset collision of the vehicle. It is believed that the vehicle-to-vehicle small offset test method is the closest to the actual traffic accident collision conditions. Therefore, the vehicle-to-vehicle collision test results are regarded as a benchmark to analyse the differences between the IIHS and NHTSA test results. The following comparative analysis of the three test methods is based on the results of three small offset tests on the same vehicle, namely the vehicle-tovehicle, IIHS and NHTSA, as is shown in Figure 11.

Figure 11 Three kinds of small offset collision tests

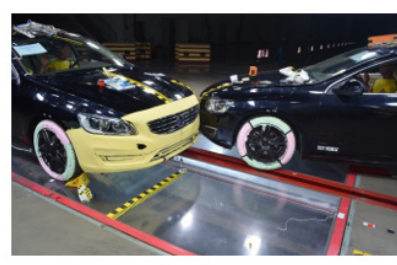

Car-to-car

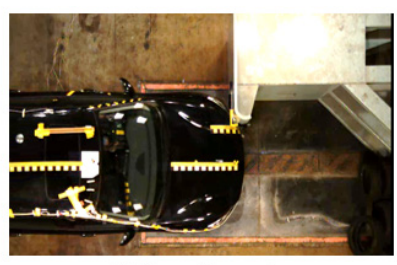

IIHS small overlap

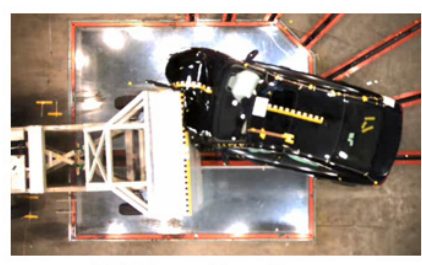

NHTSA small overlap

\subsection{Vehicle kinematics}

Compare the acceleration curves of the pillar-B on the collision side of the target vehicle in the three small offset collisions, as shown in Figure 12. In the $X$-axis acceleration curve, the peak acceleration of the IIHS test method is lower than that of the vehicle-tovehicle, but the waveform width is slightly wider. The acceleration curve of the NHTSA test method is contrary to this. The peak value is higher, but the pulse width of the waveform is slightly narrower. From the speed of the acceleration integral, the $X$-direction velocity component of the NHTSA test method is closer to that of a vehicleto-vehicle, and the gap between the speed of the IIHS method and the other two is relatively large and the overall situation is low. The $Y$-axis acceleration curve indicates that the $Y$-axis acceleration of the NHTSA test method is closer to the vehicle-to-vehicle, 
and the $Y$-axis acceleration curve of the IIHS method is similar to the $X$-direction, and the peak is lower and the pulse width is wider, but the difference between its speed integral and the $X$-direction velocity has a different trend. The $Y$-direction velocity of the IIHS method is slightly higher than that of the other two methods.

Figure 12 Comparison of vehicle body curves in three kinds of small offset collision tests

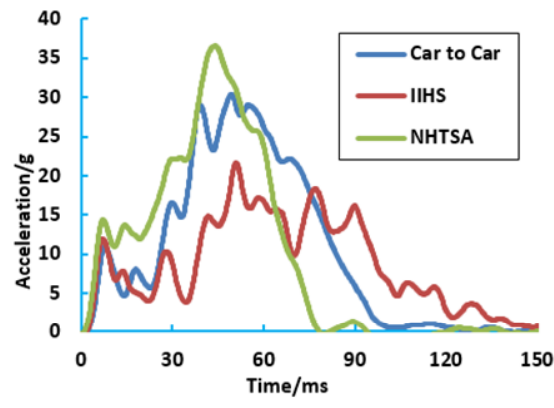

Comparison of acceleration in $X$ direction

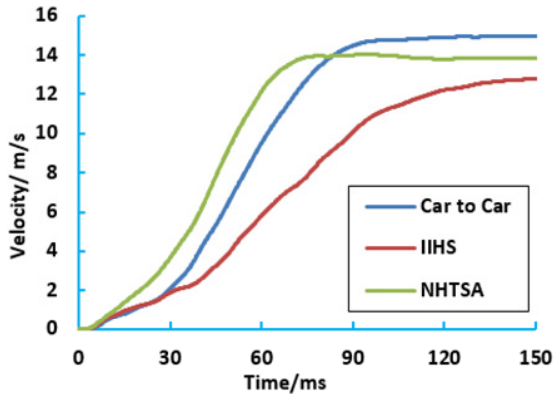

Comparison of speed in $X$-direction

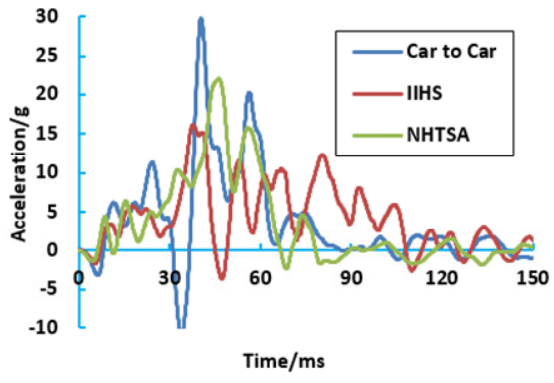

Comparison of acceleration in $Y$ direction

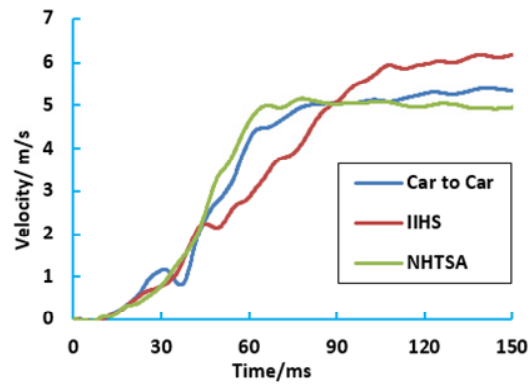

Comparison of speed in $Y$-direction

\subsection{Collision strength}

The Vehicle Pulse Index (VPI) which measures the relative strength of the collision waveform to the occupant in the vehicle uses a single-degree-of-freedom mass spring model (Tsoi and Gabler, 2015). The model consists of mass $M$, which represents the occupant, spring $k$ and slack $s$, which represent the constraint system.

Figure13 VPI calculation model

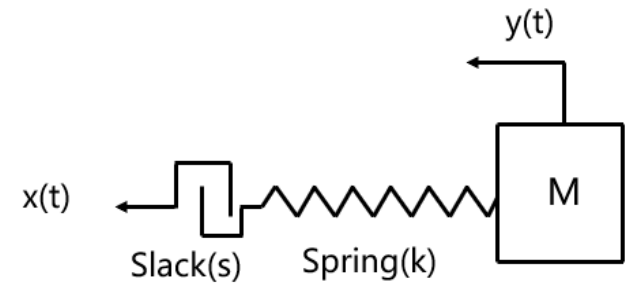


The stiffness $k$ and the slack $s$ of the restraint system can be freely defined by the user. Generally speaking, $k=2500 \mathrm{~N} / \mathrm{m} / \mathrm{kg}$ and $s=0.03 \mathrm{~m} . x(t)$ is the vehicle movement displacement function. The input of the system is usually calculated using the components of the main collision direction. $y(t)$ is the displacement function of the occupant. As the system output, its second-order differential acceleration function related to time is the VPI index. The calculation method is as follows:

$$
\begin{aligned}
& M \ddot{y}+k y=P(t) \\
& P(t)=\left\{\begin{array}{l}
0, x<s \\
k(x-s), x \geq s
\end{array}\right. \\
& V P I=\max (\ddot{y})
\end{aligned}
$$

With reference to the above methods, the VPIs of the three small offset tests are calculated and the curves are shown in Figure 14. Take its maximum value and the VPI index is $52.55 \mathrm{~g}, 32.39 \mathrm{~g}$ and $55.93 \mathrm{~g}$, respectively. As is mentioned above, it is predicted that the vehicle-to-vehicle collision strength is in direct proportion to the car deformation based on the variation of the collision velocity. However, based on the VPI index, it can also be predicted that the dummy injury of the vehicle-to-vehicle test is just in the medium among the three tests. At the same time, the dummy injury of the IIHS test is the slightest, and that of the NHTSA test is the most severe. The VPI index is vectorised and the direction of the vector refers to the $X, Y$ motion components of the car body analysed by high-speed camera imaging. The results show that the lateral motion of the IIHS small offset collision has the greatest impact on the occupants, followed by the vehicle-tovehicle and NHTSA tests. The lateral swing of the traditional $40 \%$ ODB test is the smallest, and the longitudinal VPI component is basically the same as that of the vehicleto-vehicle test.

Figure 14 VPI curve

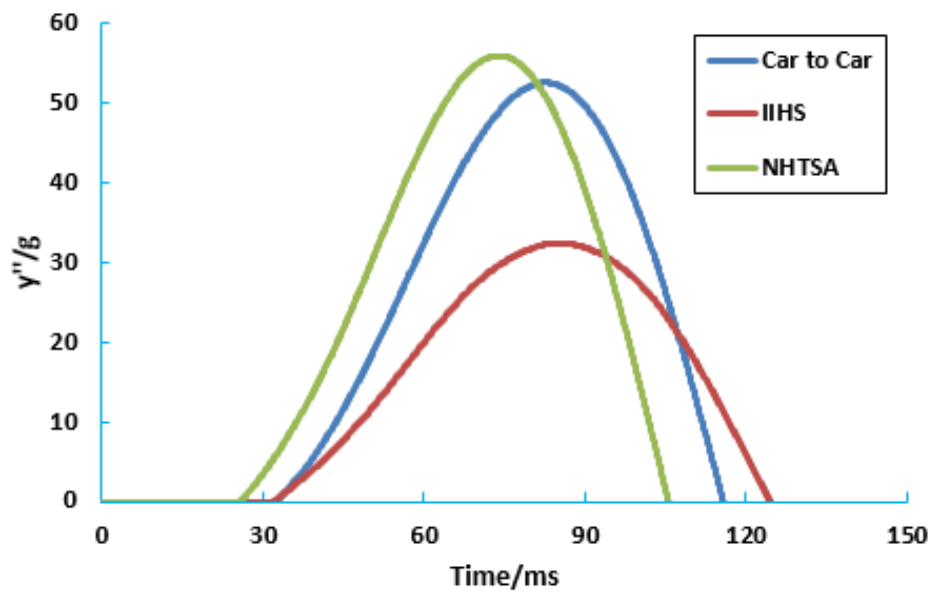




\subsection{Occupant injuries}

In the three test methods, the drivers are the closest to the collision place where the occupant space is the most invaded. Therefore, the driver is the most seriously injured. The dummy injury results at this position are used for comparative analysis. According to the order of response, NHTSA responded earliest, respectively followed by vehicle-tovehicle and IIHS. The overall performance of the three sets of acceleration curves is similar to that of VPI. Judging from the absolute value of the injury, the HIC36 of IIHS test is 87 , and the accelerated speed of the first $3 \mathrm{~ms}$ is $28.73 \mathrm{~g}$, and that of a chest at $3 \mathrm{~ms}$ is 29.55 , all of which are very small. The NHTSA and vehicle-to-vehicle tests have almost the same damage.

Table 1 Comparison of driver injury indexes in three test methods

\begin{tabular}{lccc}
\hline & $C 2 C$ & $I I H S$ & NHTSA \\
\hline HIC36 & 305 & 87 & 527 \\
Head Cum.3ms/g & 47.81 & 28.73 & 55.86 \\
Neck Upper Fx+/kN & 0.4299 & 0.01969 & 0.4332 \\
Neck Upper Fx-/kN & -0.4063 & -0.3597 & -0.1547 \\
Neck Upper Fz+/kN & 0.662 & 0.3503 & 0.9674 \\
Neck Upper My-/Nm & -6.578 & -30.18 & -5.405 \\
Chest Cum.3ms/g & 42.18 & 29.55 & 47.25 \\
Chest Dis/mm & -18.61 & -14.28 & -21.2 \\
VC/ m/s & 0.06173 & 0.02828 & 0.08104 \\
Femur force/kN & -1.549 & -0.2476 & -5.043 \\
Knee Slider/mm & 0.5106 & 1.201 & 1.222 \\
Tibia force/kN & -4.881 & -2.226 & -2.562 \\
TI & 0.9073 & 0.5029 & 0.6512 \\
\hline
\end{tabular}

\subsection{Vehicle body deformation}

In the IIHS small offset collision test, a vehicle structure evaluation is included, and the strength of the vehicle body structure is determined by measuring the deformation of key parts of the vehicle occupant compartment. With reference to the evaluation method of the IIHS vehicle body deformation, the deformation of the occupant compartment of the same vehicle model in the three collision test methods is measured and calculated, as is shown in Figure 15.

Overall, the deformation in the vehicle-to-vehicle collision pattern is the largest, especially in the dashboard, which has entered the yellow zone and the corresponding rating is acceptable. For the IIHS and NHTSA, the deformation of the occupant compartment is basically the same, only the deformation of the hinge position under the pillar A. The deformation of the IIHS method is very large, at the same level as the vehicle-to-vehicle test. 
Figure 15 Comparison of vehicle body deformation

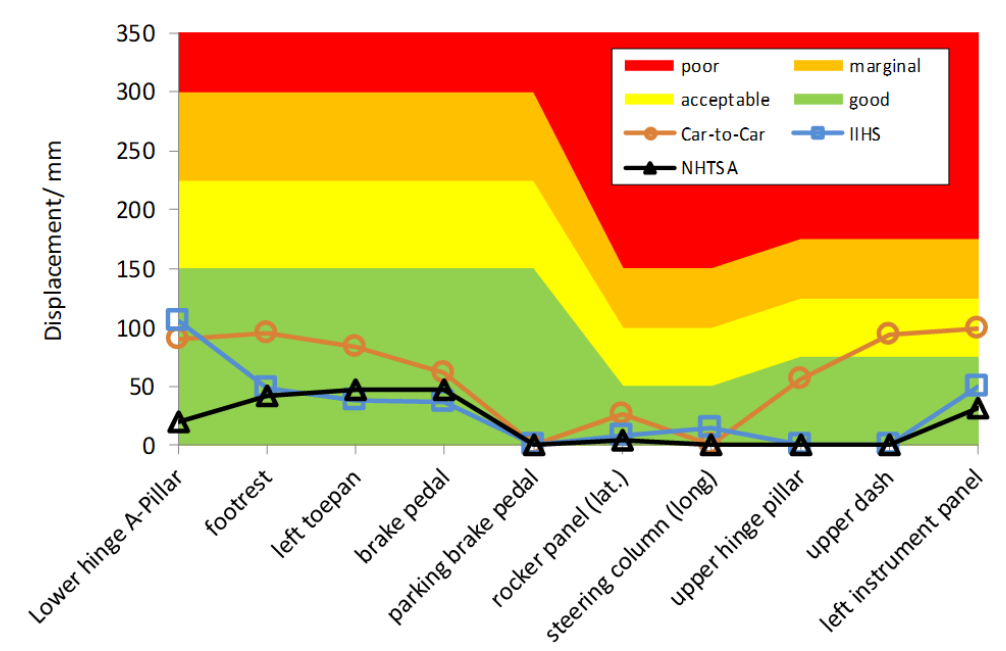

\section{Conclusions}

In this paper, two aspects of traffic accidents in small overlap front impact in China are analysed: the characteristic parameters of collision vehicles and the characteristics of occupant injuries. Small overlap front collision accounts for $27 \%$ in frontal accident in China, and the driver side (left side) accident accounts for $56 \%$. The speed of small overlap front crash was $(59.8 \pm 18.73 \mathrm{~km} / \mathrm{h})$. Serious longitudinal body deformation occurs. On the other hand, the top three AIS3 + injuries of human body segments caused by small overlap frontal collision accidents were in the head $(61 \%)$, chest $(13 \%)$ and spine $(8.7 \%)$, the proportion of the drivers suffering from serious injury is more than 6 times that in the case of a collision on the right side of the vehicle, and the rotation angle of the vehicle is less than $90^{\circ}$ after collision, the proportions of slight injury, serious injury and death in the vehicle are $30.2 \%, 9.3 \%$ and $4.7 \%$, respectively.

Regarding the comparison between the vehicle-to-vehicle, the IIHS, and the NHTSA test method, the results show that, firstly, NHTSA test method is similar to the vehicleto-vehicle test method of vehicle kinematics, while the IIHS test method is the most violent in itself. In terms of collision strength and occupant injuries, the NHTSA test method is similar to the vehicle-to-vehicle test method, and the IIHS test method is the smallest. In the aspect of vehicle body deformation, the three methods' results are at the same level, and the vehicle-to-vehicle test method is relatively large in deformation, and IIHS and NTHSA test methods are basically the same. In combination with the vehicle body acceleration, kinematics, deformation, and occupant injury factors, the NTHSA test method is more similar to the vehicle-to-vehicle test than the IIHS test method. 


\section{References}

Bean, J.D. et al. (2009) Fatalities in frontal crashes despite seat belts and air bags - Review of all CDS Cases - Model and Calendar Years 2000-2007 - 122 Fatalities, NHTSA Technical Report, Report No. DOT HS 811202.

Becky, M., Anna, M., Joe, N. and David, Z. (2015) 'Comparison of HIC and BrIC head injury risk in IIHS frontal crash tests to real-world head injuries', Proceedings of the 24th International Technical Conference on the Enhanced Safety of Vehicles (ESV), Gothenburg, Sweden.

Claudia, D.L.T., Ravi, T., Michael, G. and Andreas, S. (2017) Component Test Fixture to Improve SOI Results, SAE Technical Paper 2017-01-1466.

Dinesh, M., Dhanendra, N. and Matthew, M. (2015) Small Overlap Impact Countermeasures for Automobiles, SAE Technical Paper 2015-01-1491.

James, S. and Dan, P. (2013) 'Assessment of an oblique moving deformable barrier test procedure', Proceedings of the 22nd International Technical Conference on the Enhanced Safety of Vehicles (ESV), USA, pp.1-16.

Joyce, L., Nate, J.D., Jeff, D. and Martin, L. (2014) 'Comparison of the NHTSA research offset oblique and small overlap impact tests and the IIHS moderate and small overlap tests', SAE International Journal of Transportation Safety, Vol. 2, No. 2, pp.268-278.

Maika, K., Ning, Z., Jay, Z. and Jialou, H. (2015) 'Correlation between THOR BrIC and TBI risk from full body human model', Proceedings of the 24th International Technical Conference on the Enhanced Safety of Vehicles (ESV), Gothenburg, Sweden.

Mueller, B.C., Sherwood, C.P., Aebelaez, R.A. and Zuby, D.S. (2011) 'Comparison of hybrid III and THOR dummies in paired small overlap tests', Stapp Car Crash Journal, Vol. 55, pp.379-409.

National Highway Traffic Safety Administration (2009) Vehicle Safety Rulemaking and Research Priority Plan 2009-2011, Docket ID NHTSA-2009-0108-0023, National Highway Traffic Safety Administration, Washington, DC.

Rudd, R., Scarboro, M. and Saunders, J. (2011) 'Injury analysis of real-world small overlap and oblique frontal crashes', Proceedings of the 22nd International Technical Conference on the Enhanced Safety of Vehicles (ESV), Washington, DC.

Ryuuji, O., Taisuke, W. and Tomosaburo, O. (2015) 'Consideration of representativeness of realworld accidents and repeatability of new NHTSA oblique offset frontal impact test', Proceedings of the 24th International Technical Conference on the Enhanced Safety of Vehicles (ESV), Washington, DC, pp.1-15.

Saunders, J., Craig, M. and Parent, D. (2012) 'Moving deformable barrier test procedure for evaluating small overlap/oblique crashes', SAE International Journal of Commercial Vehicles, Vol. 5, No. 1, pp.172-195. Doi: 10.4271/2012-01-0577.

Saunders, J., Craig, M.J. and Suway, J. (2011) 'NHTSA'S test procedure evaluations for small overlap/ oblique crashes', Proceedings of the 22nd International Technical Conference on the Enhanced Safety of Vehicles (ESV), Washington, DC, pp.1-15.

Sherwood, C., Nolan, J. and Zuby, D. (2009) 'Characteristics of small overlap crashes', Proceedings of the 21st International Technical Conference on the Enhanced Safety of Vehicles (ESV), Washington, DC, pp.1-7.

Takhounts, E.G., Hasija, V., Moorhouse, K., Mcfadden, J. and Craig, M. (2013) 'Development of brain injury criteria (BrIC)', Proceedings of the 57th Stapp Car Crash Conference, Orlando, FL.

Tsoi, A.H. and Gabler, H.C. (2015) 'Evaluation of vehicle-based crash severity metrics', Traffic Injury Prevention, No. 16, pp.132-139.

United Nations Economic Commission for Europe (2015) 2015 Statistics of Road Traffic Accidents in Europe and North AMERICA, Volume LIII, New York and Geneva. 